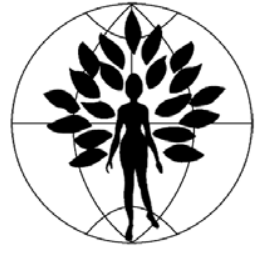

www.figo.org available at www.sciencedirect.com

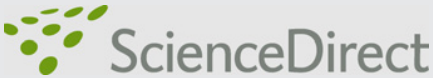

\title{
Sentinel lymph node mapping in gynecologic malignancies
}

\author{
P.V. Loar III, R.K. Reynolds*
}

Division of Gynecologic Oncology, Department of Obstetrics and Gynecology, University of Michigan, Ann Arbor, MI, USA

\author{
KEYWORDS \\ Sentinel node; \\ Gynecologic oncology; \\ Vulvar carcinoma; \\ Cervical carcinoma; \\ Endometrial carcinoma
}

\begin{abstract}
Lymph node status is the most important prognostic factor for women with vulvar, cervical and endometrial carcinoma and complete lymph node dissection has historically been an integral part of the surgical treatment of these diseases. Lymphadenectomy can be morbid for patients, who may experience wound breakdown, lymphocyst formation or chronic lymphedema, among other problems. Sentinel lymph node mapping is a newer technology that allows selective removal of the first node draining a tumor thereby allowing a potentially less aggressive procedure to be performed. Sentinel node mapping is well accepted for the management of breast carcinoma and cutaneous melanoma, and has resulted in reduced morbidity without adversely affecting survival. Sentinel node mapping is currently being investigated for treatment of gynecologic cancers. Recent studies show promise for incorporating the sentinel node mapping technique for treatment of several gynecologic malignancies.

(c) 2007 International Federation of Gynecology and Obstetrics. Published by Elsevier Ireland Ltd. All rights reserved.
\end{abstract}

\section{Introduction}

The sentinel node is defined as the first node to receive drainage in a regional node basin. If lymphatic metastasis occurs from a cancer within a given region, the sentinel node is the first site of metastasis. Should the sentinel node be free of metastasis, secondary nodes within the nodal basin should also be cancer free. Fig. 1 illustrates the concept of the sentinel node. Sentinel node mapping refers to the techniques and technologies utilized in the clinical setting to

* Corresponding author. Division of Gyn Oncology, University of Michigan Hospitals, L4000WH, 1500 East Medical Center Drive, Ann Arbor, MI 48109-0276, USA. Tel.: +1 734615 3773; fax: +1 7347647261.

E-mail address: rkr@umich.edu (R.K. Reynolds). reliably identify the sentinel node. When the sentinel node is accurately identified and the histopathology is truly negative, there is negligible risk of occult metastasis in other regional lymph nodes. Most importantly, development of validated sentinel node mapping procedures allows for the development of selective and less morbid cancer surgery in contrast to the radical, en block, resection techniques pioneered by William Halsted in the late 19th century.

The concept of the sentinel node dissection was pioneered by Cabanas and colleagues in the 1970's [1]. In a series of 80 patients with penile carcinoma, dorsal lymphatics were identified using a cut down technique after injecting blue dye into the penis, and then injecting contrast into the lymphatic to allow radiological identification of the sentinel node. Since then, others have adapted this technique for other tumors. Morton initially used blue 


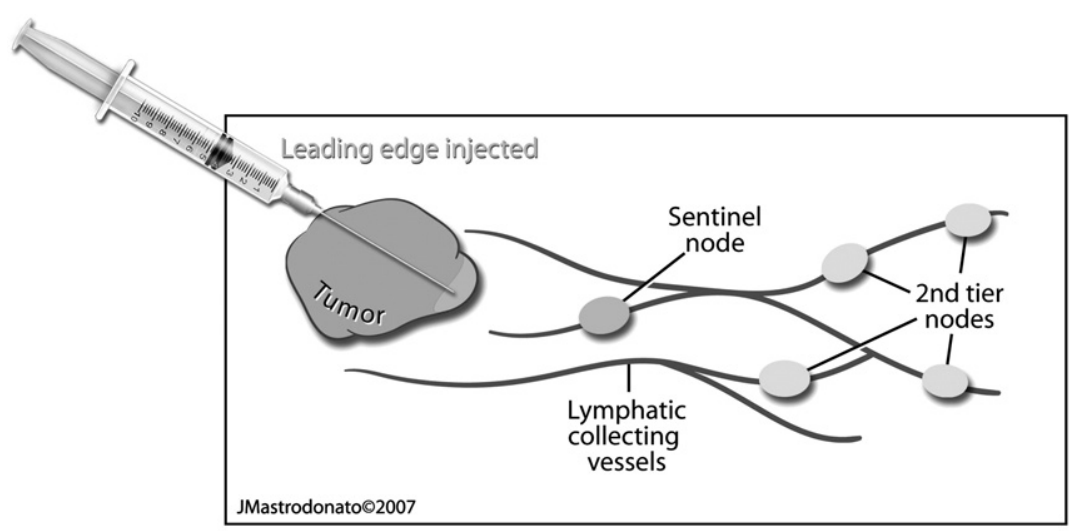

Lymphatic Mapping

Figure 1 Schematic representation of the sentinel node mapping concept. A tumor is assumed to have a single node that is the first site of metastatic spread, after which tumor will spread to other, secondary nodes (Illustration supplied by Jordan Mastrodonato).

dye injection for sentinel node mapping of melanoma and reported a true positive rate of $83 \%$, meaning that when sentinel nodes were removed and compared to the remaining nodes removed immediately afterward to complete the lymphadenectomy, the sentinel node was always positive if any other nodes were positive in $83 \%$ of cases thereby indicating the true positive rate [2]. A second approach, using radiolymphoscintigraphy involves injection of a radiolabeled colloid that is injected prior to surgery followed by a nuclear medicine scan to localize nodes. The surgeon then uses a directional gamma counter to guide the dissection. The best accuracy has been attained when both blue dye and radio radiolymphoscintigraphy are combined.

Several trials have been undertaken including the Multi-center Selective Lymphadenectomy Trials (MSLT) I \& II for treatment of melanoma. Although these trials are ongoing, preliminary results from MSLT-I show a significant survival advantage for patients who underwent wide excision followed by immediate complete lymph node dissection for a positive sentinel node biopsy compared to those who were randomized to wide excision and observation alone. Sentinel node status was the most important prognostic factor for 5-year survival in the group of patients who randomized to sentinel node biopsy. MSLT-II is comparing the value of complete lymph node dissection vs. sentinel node biopsy alone in patients with a positive SLN [3-5].

Sentinel node mapping is now an accepted procedure for treatment of both breast cancer and cutaneous melanoma. In a meta-analysis of 69 articles, Kim and colleagues reported that most institutions performing sentinel node mapping for breast cancer treatment have "abandoned the use of completion axillary lymph node dissection in patients with negative sentinel node biopsy results as experience and confidence has been gained with lymphatic mapping and sentinel node biopsy." In their report, the false negative rate was found to be inversely proportional to the number of patients included, showing a median false negative rate of $7 \%$ and illustrating the importance of experience to the accuracy of the method [6].

\section{Review of gynecologic experience}

The utility of sentinel node mapping is under investigation for treatment of vulvar, cervical and endometrial carcinoma. For each of these disease sites, lymph node status is the most important prognostic factor. Contemporary surgical treatment for each of these diseases involves a complete lymph node dissection as an integral part of the staging procedure. The probability of pelvic node metastases in cervical carcinoma ranges from $4.8 \%$ for FIGO stage la-2 to $31.4 \%$ for FIGO stage IIB and para-aortic node metastases range from $<1 \%$ to $19 \%$ for the same stages [7]. For endometrial carcinoma, the likelihood of positive pelvic nodes range from $2 \%$ to $26 \%$ for FIGO stage IA grade 1 to stage IB grade 3 , respectively and para-aortic nodes are involved $<1 \%$ to $16 \%$ for the same stages [8]. For vulvar cancer, if invasion is $<1 \mathrm{~mm}$, the incidence of nodal metastasis is essentially $0 \%$. This risk increases to $5.4 \%$ for $1-2 \mathrm{~mm}$ invasion, $8.9 \%$ for $2-$ $3 \mathrm{~mm}, 25 \%$ for $3-4 \mathrm{~mm}, 28 \%$ for $4-5 \mathrm{~mm}$ and $32 \%$ for $>5 \mathrm{~mm}$ invasion [9]. The majority of women with apparent early stage disease will have a negative metastatic work-up, and these women will have undergone an extensive surgical procedure that, in retrospect, was not needed. Most women therefore derive no benefit from a complete lymph node dissection for these cancers. With wound infection or breakdown seen in up to $85 \%$ and lymphedema in up to $23 \%$ of women with inguinal node disections, it is appealing to develop an effective surgical treatment with lower morbidity $[10,11]$.

\section{Vulvar cancer}

To date, sentinel node mapping has been most thoroughly investigated for vulvar carcinoma among the gynecologic tumors. Over the past few decades, the management of vulvar carcinoma has progressed toward less radical surgical intervention, moving away from en bloc radical vulvectomy to wide local excision or hemivulvectomy with unilateral or bilateral inguinal lymphadenectomy depending on the laterality of the tumor. The intent has been to decrease 
morbidity inherent to the procedure, including wound breakdown in about 50\%, chronic physical or functional defects in 5-25\%, lymphocyst formation in $7-28 \%$ and lymphedema in $7-19 \%$ of patients $[12,13]$. Even with the less radical procedure, inguinal lymphadenectomy still accounts for most of the perioperative morbidity. In 1978, Disaia proposed replacing the complete inguino-femoral dissection with a superficial inguinal lymphadenectomy, stating that femoral nodes should not be positive if metastases were found to be absent in the superficial nodes. Unfortunately, patients treated in this fashion experienced an unexpectedly high incidence of groin recurrence [14-17]. In 1994, Levenback published experience with sentinel node mapping for vulvar cancer using blue dye injection. A subsequent update of this study in 2001 documented accurate identification of the sentinel node in $88 \%$ of patients and $75 \%$ of groins dissected. No false negatives were reported, but a sentinel node was not identified in 2 patients. Their conclusions were that in properly selected patients, blue dye alone could identify the sentinel node in $95 \%$ of patients. Three reported risk factors for failure included midline location of the tumor, previous excisional biopsy and inexperience of the surgeon [18,19].

Ansink published experience with a similar technique but with less impressive results. Using blue dye injection, the sentinel node was found in only $56 \%$ of the 93 groins dissected and two false negatives were reported [20]. Others have noted more accurate detection rates using radiolymphoscintigraphy alone or in combination with blue dye injection. Following Decesare's initial 1997 report using injections of $99 \mathrm{~m}$ Tc-labeled colloid, multiple studies have confirmed the utility and feasibility of radiolymphoscintigraphy for vulvar cancer [21]. In a review of more than twenty studies published over the last 12 years, a total of 5 false negative sentinel nodes have been reported in nearly 450 patients and 700 groin dissections. Accurate sentinel node detection rates range from $66-100 \%$ for either blue dye or radiolymphoscintigraphy, and $91-100 \%$ if radiolymphoscintigraphy is included in the assessment. All five of the false negative studies were found in three studies: in two of the studies, the case experience of the surgeons was small ( $<25$ patients) and in one, only blue dye was used [20,22,23]. A few case reports exist in the literature describing true false negative sentinel nodes with positive nodes found distally [22,24,25]. There are no published randomized clinical trials to assess the role of sentinel node mapping for vulvar cancer. The Gynecologic Oncology Group has a large, prospective trial to assess sentinel node mapping for vulvar cancer that is currently accruing patients.

\section{Cervical cancer}

Radical hysterectomy with pelvic lymphadenectomy is a preferred treatment for early-stage, macroscopic cervical cancer. Significant postoperative morbidity includes lymphocyst formation, lymphedema, bladder dysfunction and nerve damage, all of which are at least partially associated with the lymphadenectomy. Morbidity is further exacerbated if postoperative radiation is employed.

Since the first published report by Echt in 1999 using blue dye sentinel node mapping for cervical cancer, both experience and success with the procedure have expanded considerably. The initial accurate detection rate was $23 \%$ and has since improved to a range of $80-100 \%$ when dual detection techniques were utilized with a false negative rate of $10 \%$ or lower [26].

Bats has reported an $87 \%$ accurate detection rate using both blue dye and radiolymphoscintigraphy for 25 patients with cervical cancer. The reported accuracy rate for blue dye alone was $\mathbf{8 3 . 3 \%}$ compared to $75 \%$ for radiolymphoscintigraphy. The majority of sentinel nodes were found in the interiliac regions, as expected. Of note, in 9 patients (36\%), the sentinel node was identified in an 'unusual location' outside of the inter-iliac area including the common iliac node region in 5 patients, parametrium in 2 patients and para-aortic nodes in 2 patients. These findings support their hypothesis that sentinel node mapping may improve the management of early cervical carcinoma by detecting metastasis in nodes that are not routinely removed [27].

\section{Endometrial cancer}

Sentinel node mapping of endometrial carcinoma has proven to be more problematic to develop for several reasons. First, the lymphatic drainage of the uterus is considerably more ambiguous than vulva and cervix. Second, the more focal, accessible and visible lesions that occur on the vulva or cervix are amenable to directed injection whereas endometrial lesions are often large and are always both inaccessible and not visible to the clinician for direct injection. The reported locations of sentinel nodes range from the parametrium to the para-aortic region on either side of the body. Interpreting the literature has also been complicated by the lack of standardization of injection sites. Injection sites reported include subserosal, myometrium, cervical stroma, transcervical myometrium and hysteroscopically-directed injection of endometrium. Fundal and cervical injection sites map the drainage basin of that specific segment of the uterus, but not necessarily of the tumor itself. Frumovitz states in the discussion of their recent experience with 18 women undergoing fundal injections for endometrial cancer that the injections map 'the organ rather than the cancer [28].'

An additional limitation is the timing of the injection. If the injection is to be at the fundus of the uterus, this is an intra-operative procedure in contrast to vulvar and cervical mapping where injection of the radiocolloid typically takes place 2-24 h prior to surgery thereby allowing time for collection in the nodes and subsequent imaging. Intraoperative fundal injection shortens the drainage time to minutes and could reduce the sensitivity of the test.

In studies where only blue dye was used, erratic sentinel node detection rates ranging from $0 \%$ to $100 \%$ have been reported. With the addition of radiocolloid injection, detection rates are consistently above $70 \%$. Using a combined blue dye and radiocolloid injection technique, Frumovitz reported an accurate detection rate of $31 \%$, with an increase to $80 \%$ when surgeons without experience with the procedure were excluded. Several studies have examined the use of pre-operative hysteroscopically-guided injection of the endometrium. Detection rates in these studies range from $82 \%$ to $100 \%$. Both Maccauro and Niikura confirmed that lymphatic drainage pathways of the cervix 
Table 1 Example protocol for combined blue dye and radiolymphoscintigrapghy for vulvar cancer $[18,36,37]$

- Both radiolymphoscintigraphy and blue dye are used to localize the sentinel node

- The radiocolloid used is technetium $99\left(450 \mu \mathrm{Ci}{ }^{99 m} \mathrm{Tc}\right)$ sulfur colloid (filtered to $0.2 \mu \mathrm{m}$ ) is injected intradermally in 4 sites at the periphery of the tumor $2-4 \mathrm{~h}$ pre-op

- Gamma scan is performed (see Fig. 2) and sentinel node(s) is marked

- Films are brought with the patient to the OR

- At beginning of surgical procedure, $4-8 \mathrm{ml}$ of $1 \%$ isosulfan blue dye is injected intradermally at 4 sites around the periphery of the tumor

- Incision is made over the sentinel node determined by lymphoscintigraphy

- Hand held gamma probe assists in localization of node(s)

- Blue dye is located (see Fig. 3)

- No further nodes are removed unless hand held probe identifies a second node(s) with $>10 \%$ of the sentinel node activity or $>150 \%$ of background activity

- Specimen is submitted for special processing per protocol, but NOT frozen section

- If biopsy is positive, full lymphadenectomy may be warranted depending on the clinical protocol

and the uterine corpus differed consistently, with an increased likelihood of para-aortic sentinel nodes associated with the uterine corpus $[29,30]$. This confirms the increased likelihood of false negative sentinel nodes if only the cervix is injected for endometrial cancers. A potential concern of this technique is intra-abdominal dissemination of tumor during hysteroscopy due to intrauterine distension and retrograde spill through the Fallopian tubes. Niikura addresses this issue by using only $40 \mathrm{~mm} \mathrm{Hg}$ for uterine distension which is less than the $70 \mathrm{~mm} \mathrm{Hg}$ usually needed for tubal distension. Second, hysteroscopy was performed on the day of definitive surgery, thereby decreasing the time for possible extruded cells to remain in the abdomen prior to irrigation. Niikura reported one case of positive cytology and no intraperitoneal spillage of blue dye was noted in any patient.

\section{Technique}

A number of methods have been reported for blue dye and radiolymphoscintigraphy techniques. Typically, a blue dye, a radiocolloid, or both, is injected into the tissue surrounding the tumor. Blue dyes used include either patent blue or isosulfan blue. The radioactive tracers most often used are either Technetium-99m labeled albumin or sulfur colloid. Radiocolloid is injected usually $2-4$ h preoperatively if ${ }^{99 \mathrm{~m}} \mathrm{Tc}$ sulfur colloid is used and on pre-op day 1 if ${ }^{99 m}$ Tc albumin is used, although a number of protocol variations have been reported. Vulvar lesions are typically injected at the junction of the tumor and normal skin in varying patterns, often dependent upon the location of the lesion. In cases of cervical malignancy, the cervical stroma is injected circumferentially. Peri-tumoral injection is preferred, as it should allow the tracer to follow the same path as the lymphatic drainage of the lesion itself. In the case of endometrial carcinomas the site(s) of injection are not as well defined. If a radioisotope is employed, a preoperative radiolymphoscintigram is performed on either the day prior to or the day of surgery to aid in localization of the sentinel node(s). For patients with vulvar cancer, the skin overlying the sentinel node is marked using the radiolymphoscintigram, allowing for smaller, more selective incisions. Blue dye injection typically takes place immediately prior to surgical incision. Table 1 provides a typical protocol for combined blue dye and radiolymphoscintigraphy.

In the operating room, dissection of the sentinel node is performed first in most cases. In vulvar procedures using the combined blue dye and radiolymphoscintigraphy technique, the superficial inguinal nodes are examined with a handheld, directional gamma probe prior to and after incision to guide dissection in conjunction with visual inspection for the presence of blue lymphatic channels and nodes after the incision is made. If a preoperative radiolymphoscintigram was performed, this image is used to guide the site and size of the incision and to localize the sentinel node. In cases of cervical or endometrial carcinoma, the lymphatic evaluation

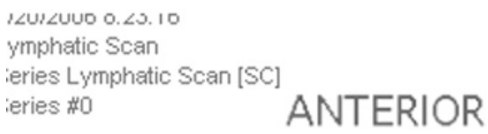

ANTERIOR
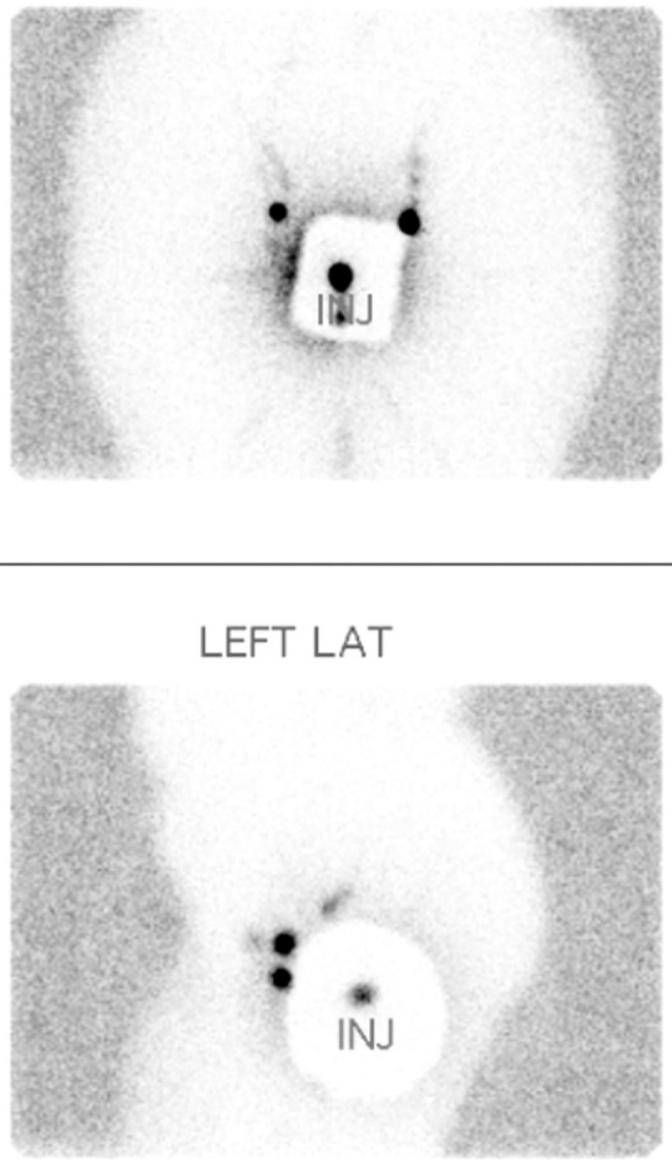

Figure 2 Radiolymphoscintigram showing 'hot' nodes. The center of the image is blocked to prevent washout of the image by the high level of radioactivity at the site of injection on the vulva. This image is utilized by the surgeon to plan the site of incisions in order to minimize the extent of the dissection. 


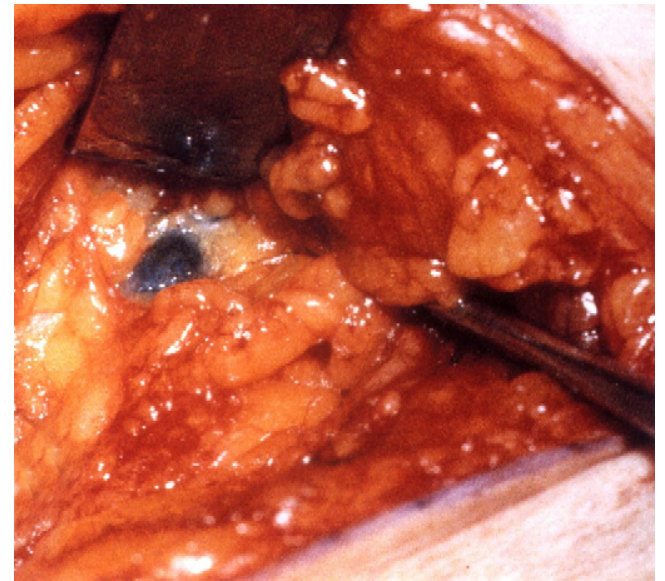

Figure 3 Photograph of sentinel node displaying uptake of blue dye. The blue color, either alone, or in conjunction with use of radiocolloid injection and radiolymphoscintography, allows the surgeon to identify the sentinel node which can then be selectively excised.

is performed in a similar fashion utilizing either laparoscopy or laparotomy. A laparoscopic gamma probe is employed for the minimally invasive procedures. Once the sentinel node is localized and excised with as little disruption as possible to surrounding tissues, the directional gamma counter is used to assess for background radiation that indicates if the correct node has been removed or if there is another sentinel node. The background radiation count should not exceed $10 \%$ of the count from the sentinel node. Nodes are usually re-examined with the probe ex vivo to confirm radioactivity, and the lymphadenectomy site is reassessed to exclude residual radioactivity. Sentinel nodes are then sent for pathological evaluation as separate specimens.

\section{Pathology}

The histopathologic examination of a sentinel node is a crucial step for accurate diagnosis and the results will dictate if further surgical intervention such as completion lymphadenectomy is necessary. Frozen section was originally favored as the determinant of whether completion lymphadenectomy was warranted. However, false negative rates as high as $70 \%$ were reported for frozen sections, and this step has been omitted from sentinel node evaluation in most centers. Typically, routine paraffin embedding and hematoxylin and eosin staining is performed. In cases with micrometastases, serial step sectioning and immunohistochemistry studies specific to the tumor type have been reported to greatly increase accuracy of diagnosis and to minimize the likelihood of a false negative report. This technique has resulted in an increase in rate of positive node detection reported in a number of studies [31-35]. De Hullu was one of the first to publish experience with ultrastaging in 59 patients with vulvar carcinoma. This technique detected 4 additional metastatic lymph nodes out of 102 (4\%) originally found to be negative on routine histological examination. A number of authors have reported similar findings, with increases in the node positivity rate of up to $5.1 \%$.

\section{Conclusion}

The presence of lymph node metastasis remains the most important prognostic factor for women with vulvar, cervical and endometrial carcinoma, but complete lymphadenectomy for these patients can be morbid and costly. The ability to detect the presence of lymphatic tumor spread by accurately assessing the sentinel node in the lymphatic drainage basin has been shown to be feasible for many cancer types, including vulvar and cervical cancers, and is under development for endometrial cancer. Once prospective studies have been completed to assess for differences in outcome, sentinel node mapping will allow for selective removal of nodes at risk, while sparing other nodes and reducing operative morbidity substantially. Sentinel node mapping is already accepted for the management of breast cancer and cutaneous melanoma. For gynecologic cancers, the results are promising, particularly for vulvar cancer. Until any of several large, ongoing, multicenter trials are completed, this technological advancement will be considered promising, but not yet a standard of care for treatment of women with gynecologic malignancies. Given the collective interest in the less morbid treatment for patients with cancer, sentinel node mapping deserves continued development.

\section{References}

[1] Cabanas RM. An approach for the treatment of penile carcinoma. Cancer 1977;39(2):456-66.

[2] Morton DL, Wen DR, Wong JH, Economou JS, Cagle LA, Storm FK, et al. Technical details of intraoperative lymphatic mapping for early stage melanoma. Arch Surg 1992;127(4):392-9.

[3] Morton DL, Cochran AJ, Thompson JF, Elashoff R, Essner R, Glass EC, et al. Multicenter Selective Lymphadenectomy Trial Group. Sentinel node biopsy for early-stage melanoma: accuracy and morbidity in MSLT-I, an international multicenter trial. Ann Surg 2005;242(3):302-11.

[4] EsSLNer R. Sentinel lymph node biopsy and melanoma biology. Clin Cancer Res 2006;12(7 Pt 2):2320s-5s.

[5] McMasters KM, Noyes RD, Reintgen DS, Goydos JS, Beitsch PD, Davidson BS, et al. Lessons learned from the Sunbelt Melanoma Trial. J Surg Oncol 2004;86(4):212-23.

[6] Kim T, Giuliano AE, Lyman GH. Lymphatic mapping and sentinel lymph node biopsy in early-stage breast carcinoma: a metaanalysis. Cancer 2006;106(1):4-16.

[7] Morrow CP, Curtin JP, Townsend DE. Synopsis of gynecologic oncology. Fourth ed. New York: Churchill Livingstone; 1993.

[8] Creasman WT, Morrow CP, Bundy BN, Homesley HD, Graham JE, Heller PB. Surgical pathologic spread patterns of endometrial cancer. A Gynecologic Oncology Group Study. Cancer 1987;60(8 Suppl):2035-41.

[9] Oonk MHM, Hollema H, DeHullu JA, van der Zee AGJ. Prediction of lymph node metastases in vulvar cancer: a review. Int J Gynecol Cancer 2006;16:963-71.

[10] Martimbeau PW, Kjorstad KE, Kolstad P. Stage IB carcinoma of the cervix, the Norwegian Radium Hospital, 1968-1970: results of treatment and major complications. I. Lymphedema. Am J Obstet Gynecol 1978;131(4):389-94.

[11] Podratz KC, Symmonds RE, Taylor WF, Williams TJ. Carcinoma of the vulva: analysis of treatment and survival. Obstet Gynecol 1983;61(1):63-74.

[12] Burrell MO, Franklin III EW, Campion MJ, Crozier MA, Stacy DW. The modified radical vulvectomy with groin dissection: an eightyear experience. Am J Obstet Gynecol 1988;159(3):715-22. 
[13] Morrow CP. Surgery for vulvar neoplasia. Gynecologic Cancer Surgery. New York: Churchill Livingstone; 1996. p. 831.

[14] De Hullu JA, Hollema H, Lolkema S, Boezen M, Boonstra H, Burger MP, et al. Vulvar carcinoma. The price of less radical surgery. Cancer 2002;95(11):2331-8.

[15] De Hullu JA, Oonk MH, van der Zee AG. Modern management of vulvar cancer. Curr Opin Obstet Gynecol 2004;16(1):65-72.

[16] DiSaia PJ, Creasman WT, Rich WM. An alternate approach to early cancer of the vulva. Am J Obstet Gynecol 1979;133(7):825-32.

[17] Gordinier ME, Malpica A, Burke TW, Bodurka DC, Wolf JK, Jhingran A, et al. Groin recurrence in patients with vulvar cancer with negative nodes on superficial inguinal lymphadenectomy. Gynecol Oncol 2003;90(3):625-8.

[18] Levenback C, Burke TW, Gershenson DM, Morris M, Malpica A, Ross MI. Intraoperative lymphatic mapping for vulvar cancer. Obstet Gynecol 1994;84(2):163-7.

[19] Levenback C, Coleman RL, Burke TW, Bodurka-Bevers D, Wolf JK, Gershenson DM. Intraoperative lymphatic mapping and sentinel node identification with blue dye in patients with vulvar cancer. Gynecol Oncol 2001;83(2):276-81.

[20] Ansink AC, Sie-Go DM, van der Velden J, Sijmons EA, de Barros Lopes A, Monaghan JM, et al. Identification of sentinel lymph nodes in vulvar carcinoma patients with the aid of a patent blue $\mathrm{V}$ injection: a multicenter study. Cancer 1999; 86(4):652-6.

[21] Decesare SL, Fiorica JV, Roberts WS, Reintgen D, Arango H, Hoffman MS, et al. A pilot study utilizing intraoperative lymphoscintigraphy for identification of the sentinel lymph nodes in vulvar cancer. Gynecol Oncol 1997;66(3):425-8.

[22] Boran N, Kayikcioglu F, Kir M. Sentinel lymph node procedure in early vulvar cancer. Gynecol Oncol 2003;90(2):492-3

[23] Merisio C, Berretta R, Gualdi M, Pultrone DC, Anfuso S, Agnese $G$, et al. Radioguided sentinel lymph node detection in vulvar cancer. Int J Gynecol Cancer 2005;15(3):493-7.

[24] Hakam A, Nasir A, Raghuwanshi R, Smith PV, Crawley S, Kaiser $\mathrm{HE}$, et al. Value of multilevel sectioning for improved detection of micrometastases in sentinel lymph nodes in invasive squamous cell carcinoma of the vulva. Anticancer Res 2004;24 (2C):1281-6.

[25] Raspagliesi F, Ditto A, Fontanelli R, Maccauro M, Carcangiu ML, Parazzini $F$, et al. False-negative sentinel node in patients with vulvar cancer: a case study. Int J Gynecol Cancer 2003;13 (3):361-3.

[26] Echt ML, Finan MA, Hoffman MS, Kline RC, Roberts WS, Fiorica JV. Detection of sentinel lymph nodes with lymphazurin in cervical, uterine, and vulvar malignancies. South Med J 1999;92 (2):204-8.

[27] Bats AS, Clément D, Larousserie F, Lefrère-Belda MA, Faraggi $M$, Froissart $M$, et al. Sentinel lymph node biopsy improves staging in early cervical cancer. Gynecol Oncol 2007;105(1): 189-193.

[28] Frumovitz M, Bodurka DC, Broaddus RR, Coleman RL, Sood AK, Gershenson DM, et al. Lymphatic mapping and sentinel node biopsy in women with high-risk endometrial cancer. Gynecol Oncol 2007;104(1):100-3.

[29] Maccauro M, Lucignani G, Aliberti G, Villano C, Castellani MR, Solima E, et al. Sentinel lymph node detection following the hysteroscopic peritumoural injection of $99 \mathrm{mTc}$-labelled albumin nanocolloid in endometrial cancer. Eur J Nucl Med Mol Imaging 2005;32(5):569-74.

[30] Niikura H, Okamura C, Utsunomiya H, Yoshinaga K, Akahira J, Ito $\mathrm{K}$, et al. Sentinel lymph node detection in patients with endometrial cancer. Gynecol Oncol 2004;92(2):669-74.

[31] de Hullu JA, Hollema H, Piers DA, Verheijen RH, van Diest PJ, Mourits $M J$, et al. Sentinel lymph node procedure is highly accurate in squamous cell carcinoma of the vulva. J Clin Oncol 2000;18(15):2811-6.

[32] Di Stefano AB, Acquaviva G, Garozzo G, Barbic M, Cvjeticanin $B$, Meglic L, et al. Lymph node mapping and sentinel node detection in patients with cervical carcinoma: a 2-year experience. Gynecol Oncol 2005;99(3):671-9.

[33] Louis-Sylvestre C, Evangelista E, Leonard F, Itti E, Meignan M, Paniel BJ. Sentinel node localization should be interpreted with caution in midline vulvar cancer. Gynecol Oncol 2005;97 (1):151-4.

[34] Rob L, Robova H, Pluta M, Strnad P, Kacirek J, Skapa P, et al. Further data on sentinel lymph node mapping in vulvar cancer by blue dye and radiocolloid Tc99. Int J Gynecol Cancer 2007;17 (1):147-53.

[35] Silva LB, Silva-Filho AL, Traiman P, Triginelli SA, de Lima CF, Siqueira CF, et al. Sentinel node detection in cervical cancer with (99m)Tc-phytate. Gynecol Oncol 2005;97(2):588-95.

[36] Fiorica J, Grendys E, Hoffman M. Intraoperative radiolocalization of the sentinel node in patients with vulvar cancer. Oper Tech Gynecolog Surg 2001;6:27.

[37] Moore DH, Koh WJ, McGuire WP, Wilkinson EJ. Vulva. In: Hoskins WJ, Perez CA, Young RC, Barakat R, Markman M, Randall $M$, editors. Principles and practice of gynecologic oncology. 4th edition. Philadelphia: Lippincott Williams \& Wilkins; 2005. 